\title{
Engineering CRISPR guide RNA riboswitches for in vivo applications
}

\section{Roberto Galizi}

r.galizi11@imperial.ac.uk

\section{Alfonso Jaramillo $2,3,4$}

alfonso.Jaramillo@synth-bio.org

${ }^{1}$ Department of Life Sciences, Imperial College London, London, United Kingdom

${ }^{2}$ Warwick Integrative Synthetic Biology Centre (WISB) and School of Life Sciences, University of Warwick, CV4 7AL Coventry, United Kingdom

${ }^{3}$ ISSB, CNRS, Univ Evry, CEA, Université Paris-Saclay, 91025 Evry, France

${ }^{4}$ Institute for Integrative Systems Biology (I2SysBio), University of Valencia-CSIC, 46980 Paterna, Spain

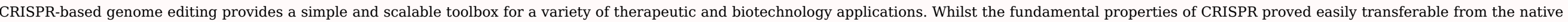

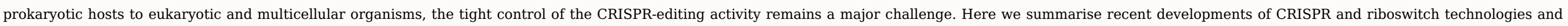

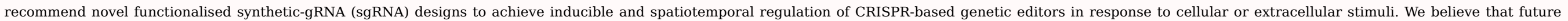
advances of these tools will have major implications for both basic and applied research, spanning from fundamental genetic studies and synthetic biology to genetic editing and gene therapy

Current Opinion in Biotechnology 2018, 55:xx-yy

This review comes from a themed issue on Analytical biotechnology

Edited by Saulius Klimasauskas and Linas Mazutis

https://doi.org/10.1016/j.copbio.2018.08.008.007

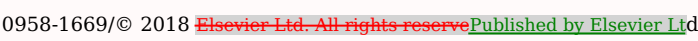

\section{Introduction}

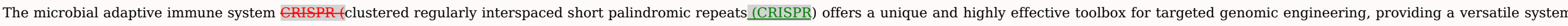

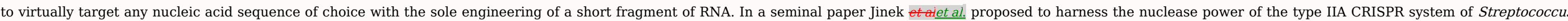

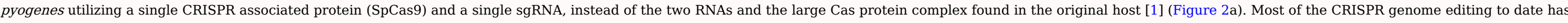

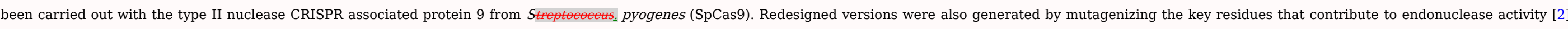

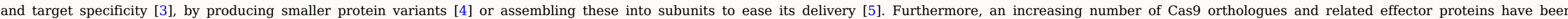

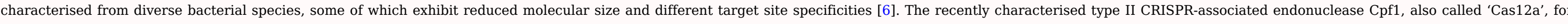

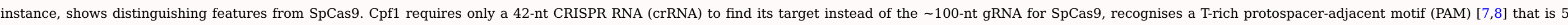

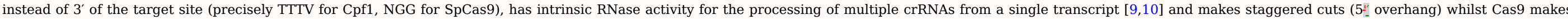

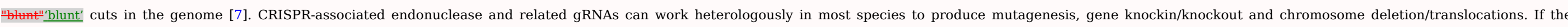

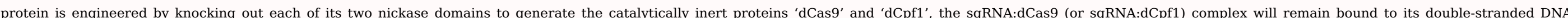




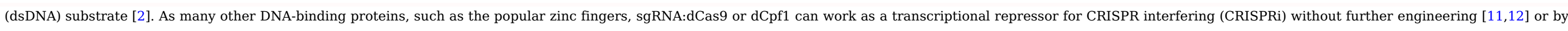

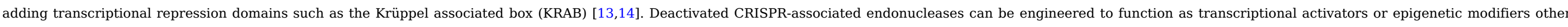

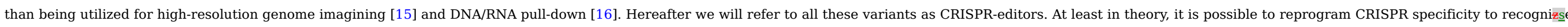

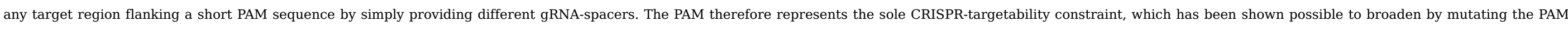

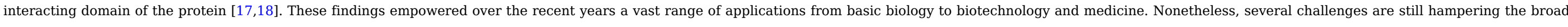

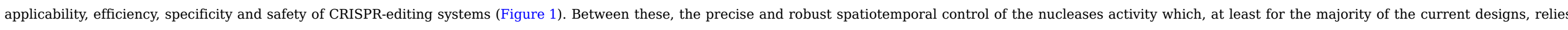

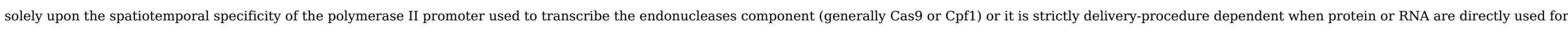

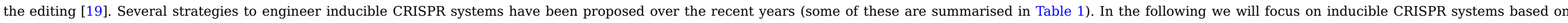
riboswitch regulation by giving an overview of the existing tools, propose new designs and highlight some of the current and foreseen applications.

A)

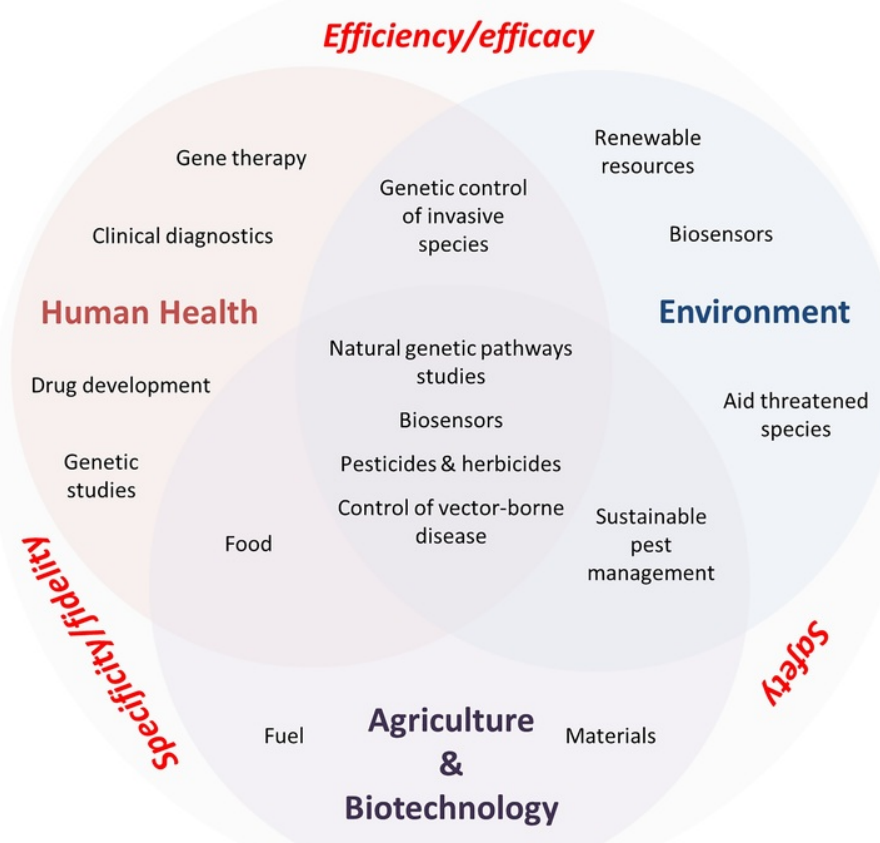

B)

\section{Biotechnology}

\section{$\Lambda$ \\ Challenges Target site selection-Spatiotemporal activity - Resistance- Incidence of HDR
Off-targets- Delivery-Confinement-Containment}

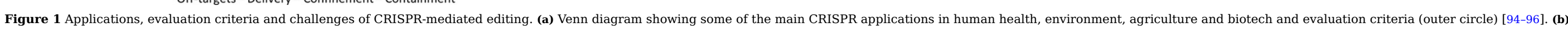

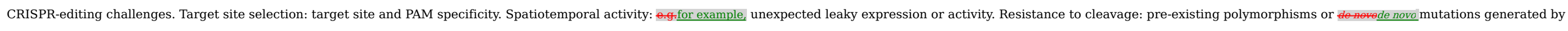

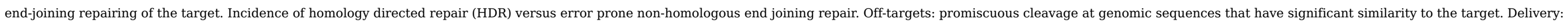

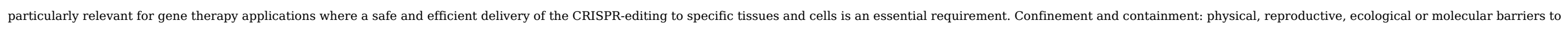
limit CRISPR-editing to the target organisms o species $[93,97,98]$. 


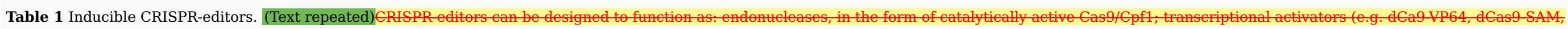

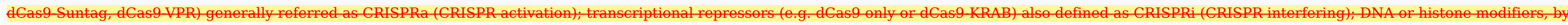
fusing dCas9 or dCpf1 proteins to histone demethylases (LSD1), histone acetyltransferases (p300), DNA methyltransferases (DNMT3a) or DNA demethylases (TET1) [95,98,99] alt-text: Table 1

\section{CRISPR-editors}

Endonucleases, activators, repressors, modifiers

Inducible CRISPR systems

$$
\text { Types }
$$

Cis-regulatory elements

RNA transcript interactions (riboregulators)

Split system

\section{Allosteric system}

Combinatorial system

Inhibitory molecules or III responsive)

ncRNA

Riboswitches

Toehold switches

Switch-gRNA

Intein-mediated

Rapamycin-mediated

Magnet-mediated

Cryptochrome-mediated

Dimerization

Anti-Cas9 proteins

Examples

Promoters, enhancers, insulators and silencers (polymerase I

Conformational change of Cas9/Cpf1

Inducible promoters (tetracycline, doxycycline, etc.)

Recombinase-inducible (Cre)

Anti-gRNA

gRNA mimics

\section{Regulation}

Exogenous

Endogenous

\section{Source}

\section{Biological}

Synthetic or heterologous/pathogenic RNA, DNA, proteins (e.g transcription factors)

Chemical

Drugs, small molecules

Physical

Light, $\mathrm{pH}$, temperature

Organism/specie-specific
RNA (mRNA or ncRNA)

$D N A$, proteins, $p H$, temperature

Type

Tissue/cell-specific

Cellular state-specific

e.g. quiescent/active, stress response,

mutations)

(e.g. vector-specific for gene drive

applications)

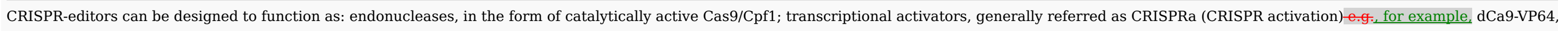

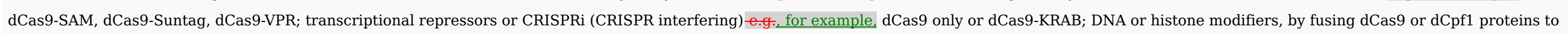

histone demethylases (LSD1), histone acetyltransferases (p300), DNA methyltransferases (DNMT3a) or DNA demethylases (TET1)[95,98,99].

\section{Riboregulation and riboswitches: current designs and applications}

\section{Natural and synthetic riboswitches}

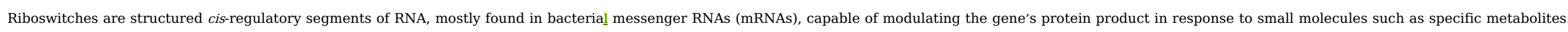

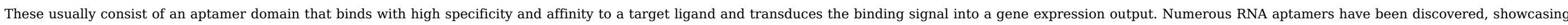




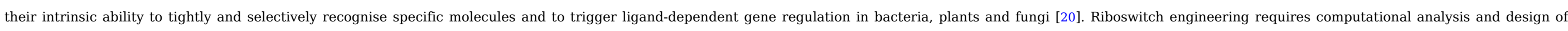

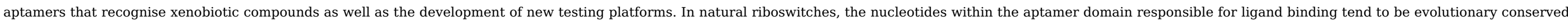

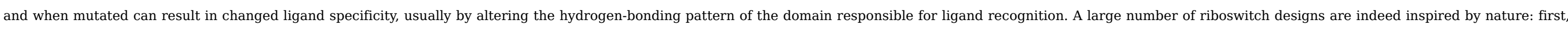

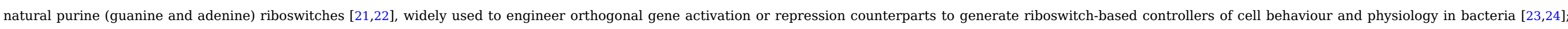

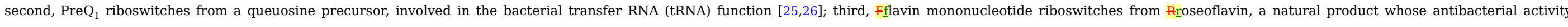

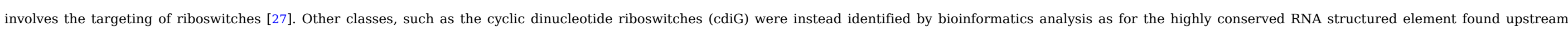
'GEMM motif' (genes for environment, membranes and motility) [28].

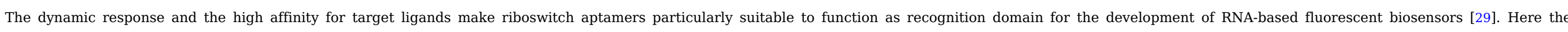

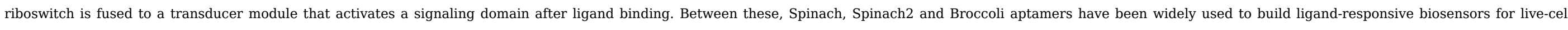

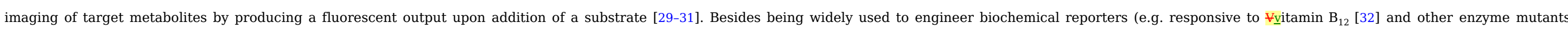

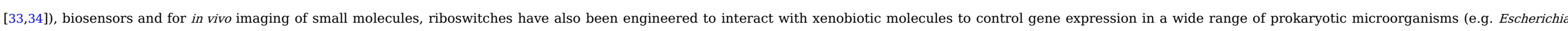

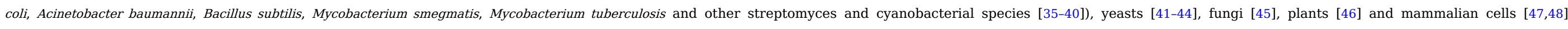

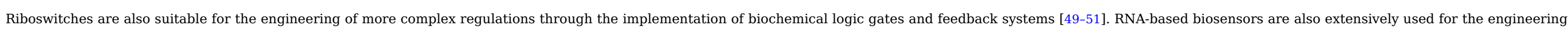
of metabolic pathways to optimise the production of small molecules such as industrial and fuel compounds [52], or for the sensing of intracellular behaviours and environmental conditions [53].

\section{RNA-responsive riboswitches}

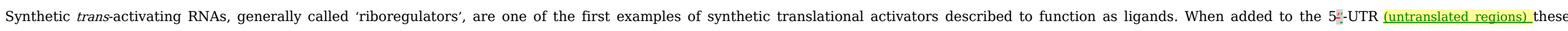

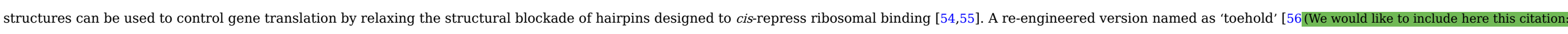

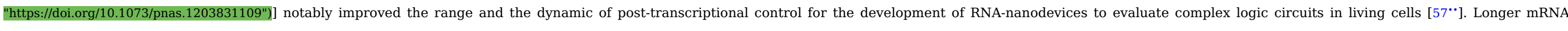

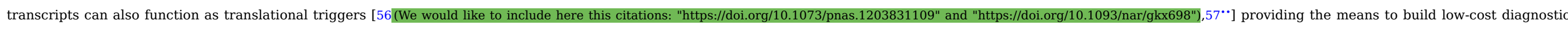
platforms based on toehold designs [58].

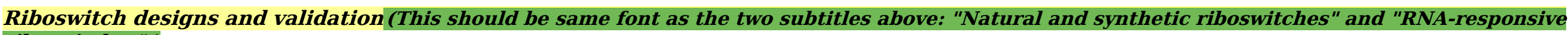

\section{riboswitches")}

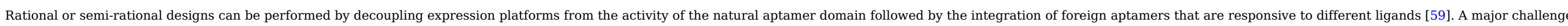

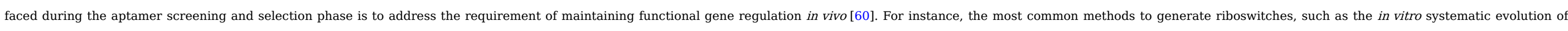

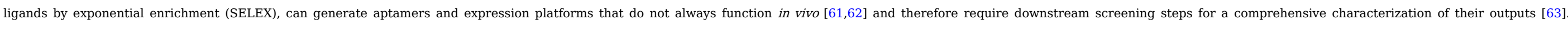

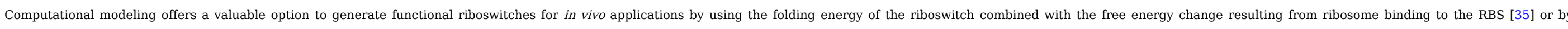

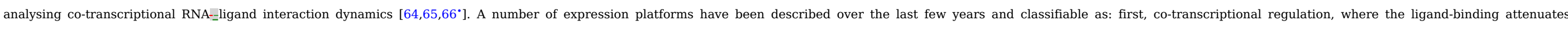

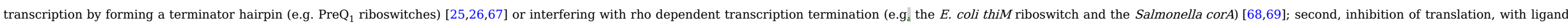

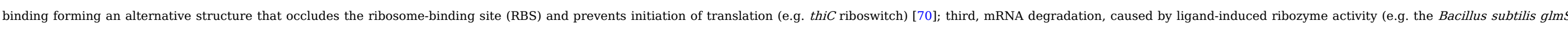

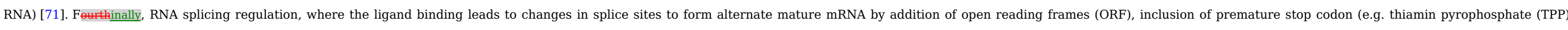

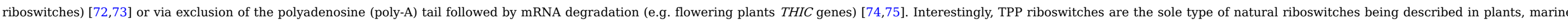

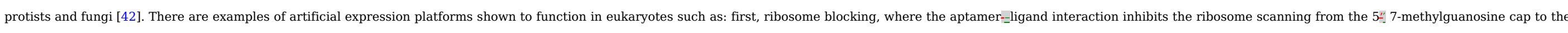

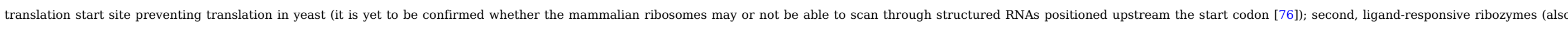

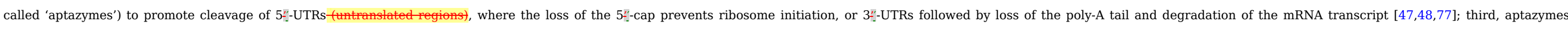
associated to micro RNA (miRNA) precursors [78,79] or orthogonal tRNAs for unnatural amino acid incorporation and studies of ribosome function (e.g. T box riboswitches) [37].

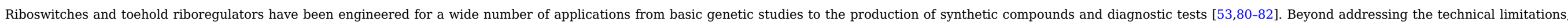




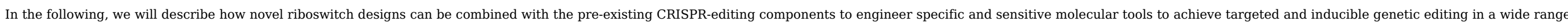
of prokaryotic and eukaryotic hosts. We believe that these technologies could be potentially transferrable to any CRISPR-based application.

\section{CRISPR gRNA riboswitches: new designs and future applications}

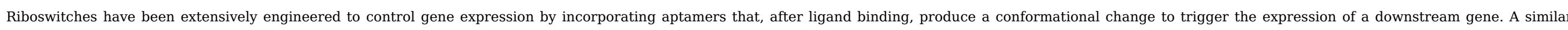

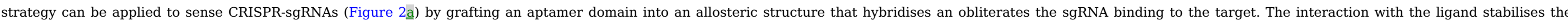

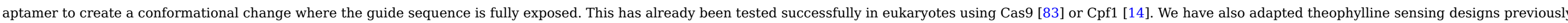

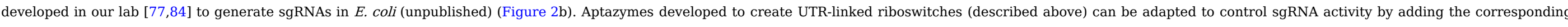

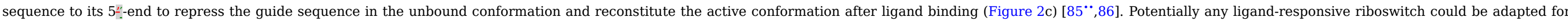

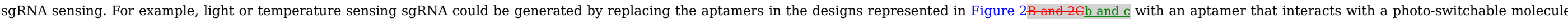

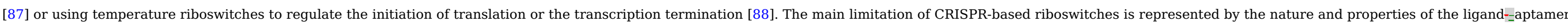

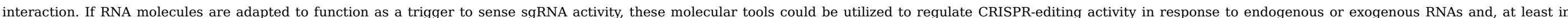
theory, could be used for any application and organism where specific spatiotemporal or conditional regulation may be required.

A)

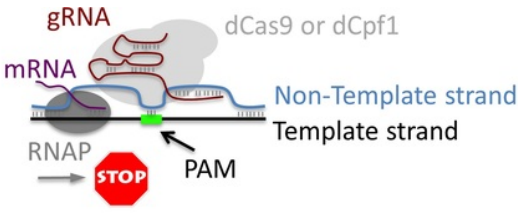

Obliterated

B) terminator Aptamer
$O R$

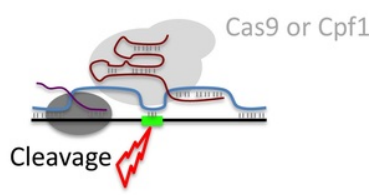

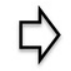

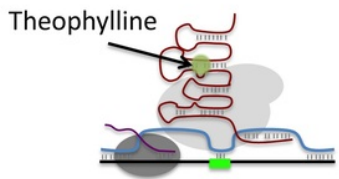

Theophylline stabilises ribozyme

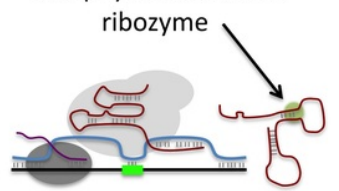

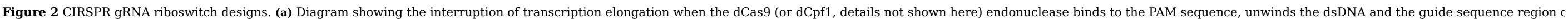

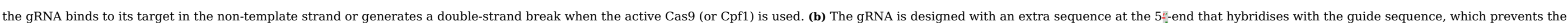

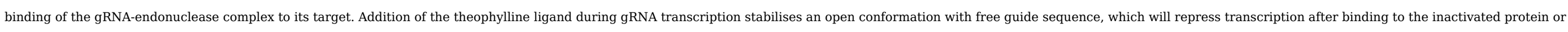

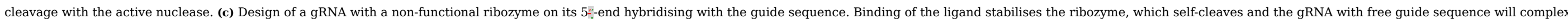

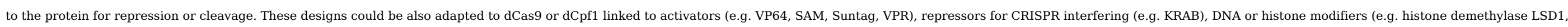
histone acetyltransferase p300, DNA methyltransferase DNMT3a, DNA demethylase TET1). 


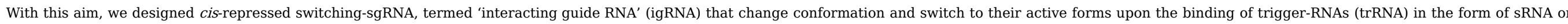

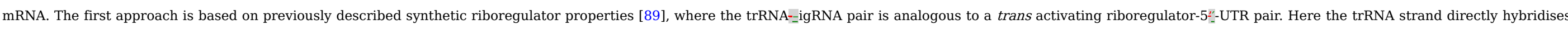

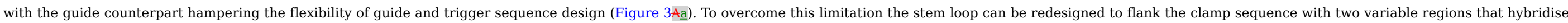

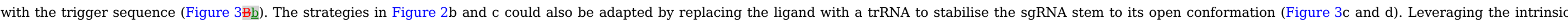

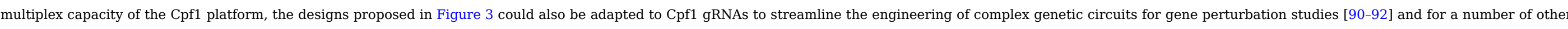

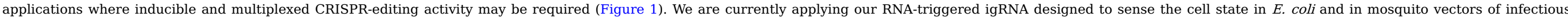
diseases to activate CRISPR-Cas endonucleases in response to tissue specific endogenous transcripts.

A)

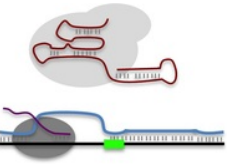

B)

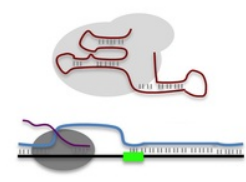

C) Obliterated terminator

D)
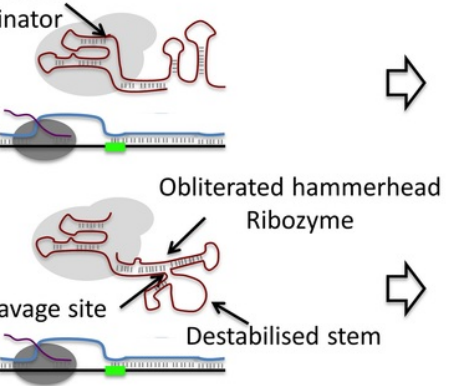

¿

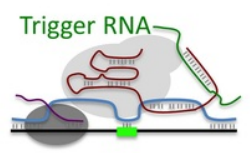

ᄃ)

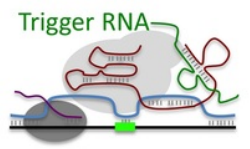

Stabilised $\sim$
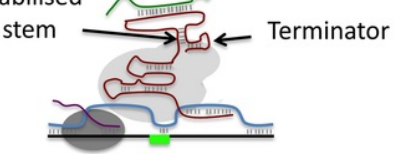

Trigger RNA stabilises stabilised stem

self-cleaving ribozyme stabilised stem

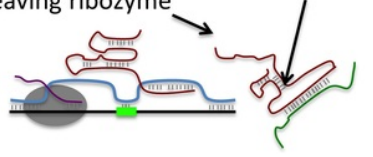

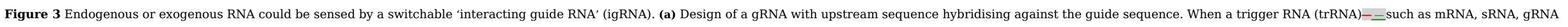

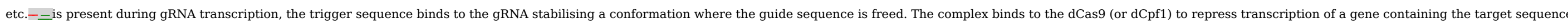

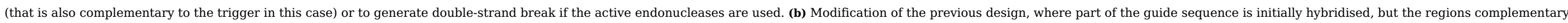

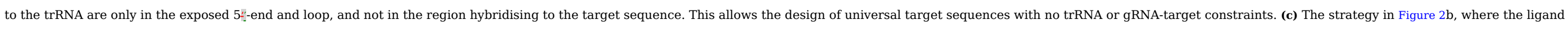

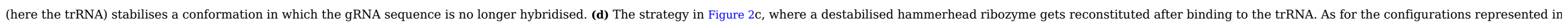
Figure 2, also these designs could be adapted to any other type of Cas9 or Cpf1 CRISPR-editor that may function as inducible endonuclease, transcriptional activator or repressors, DNA or histone modifier.

\section{alt-text: Figure 3}

\section{Conclusions}

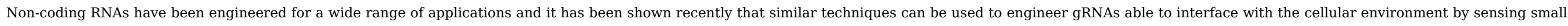

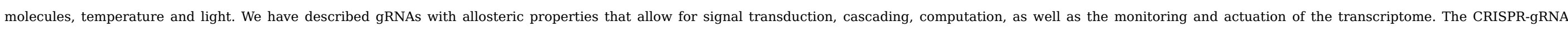

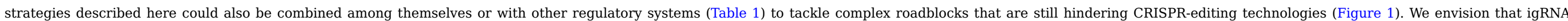




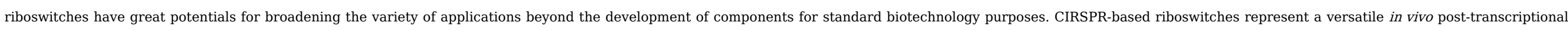

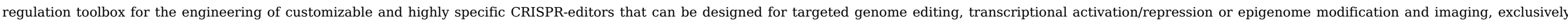

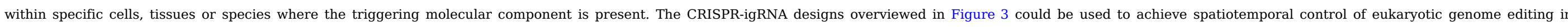

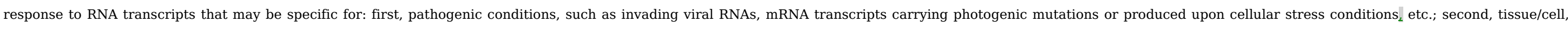

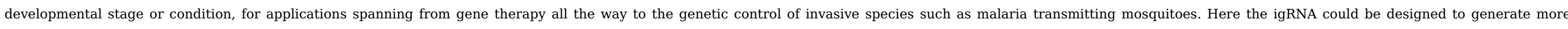

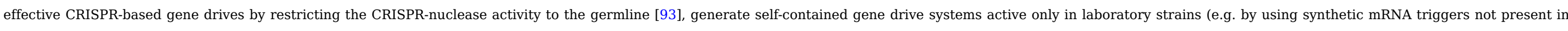
wild-type species) or even to engineer suicidal transgenes that respond to specific adverse gene mutations (such as the ones causing insecticide resistance in the mosquitoes).

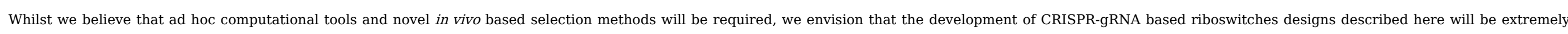
valuable to tackle most of the challenges that are still impeding the deployment of CRISPR-mediated genome editing and engineering.

\section{Conflict of interest statement}

Nothing declared.

\section{References and recommended reading}

Papers of particular interest, published within the period of review, have been highlighted as:

$$
\text { - of special interest }
$$

.. of outstanding interest

\section{Acknowledgements}

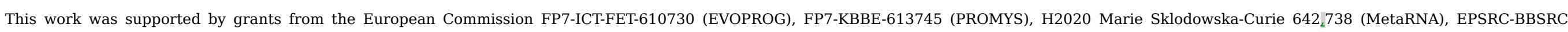
BB/M017982/1 (WISB center), BBSRC grant EVO-ENGINE BB/P020615/1 and the startup allocation from the School of Life Sciences.

\section{References}

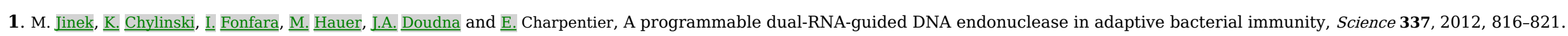

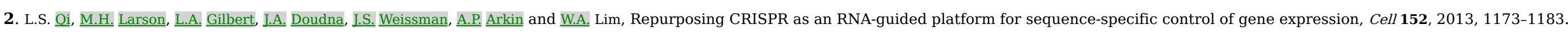

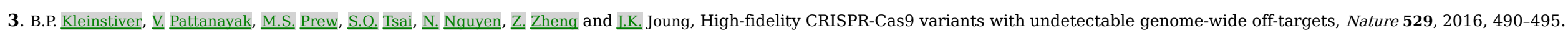

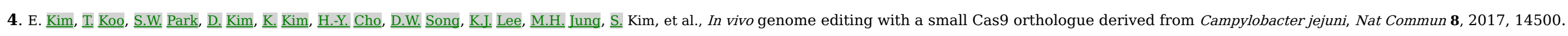

5. B. Zetsche, $\underline{\text { S.E. }}$ Volz and E. Zhang, A split-Cas9 architecture for inducible genome editing and transcription modulation, Nat Biotechnol 2015, https://doi.org/10.1038/nbt.3149.

6. $\mathrm{A}_{-}$Cebrian-Serrano and $\mathrm{B}_{\Perp}$ Davies, CRISPR-Cas orthologues and variants: optimizing the repertoire, specificity and delivery of genome engineering tools, Mamm Genome 28, $2017,247-261$.

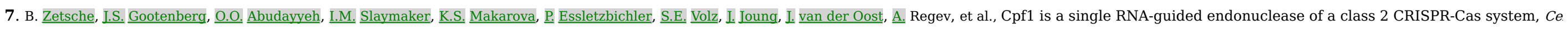
163, 2015, 759-771.

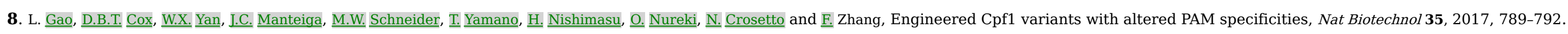

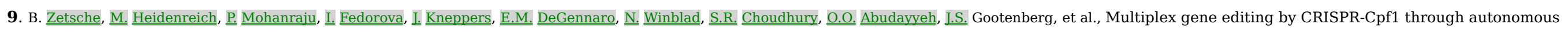
processing of a single crRNA array, Nat Biotechnol 35, 2017, 31-34. 


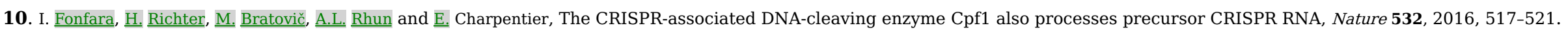

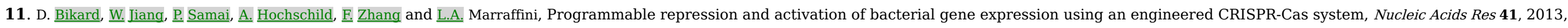
7429-7437.

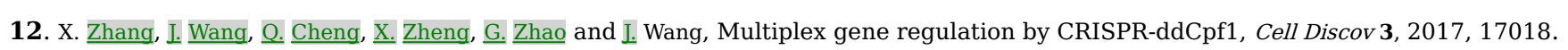

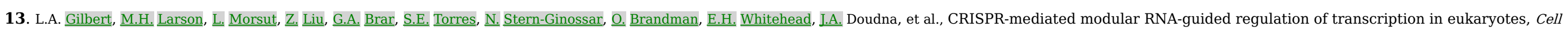
154, 2013, 442-451.

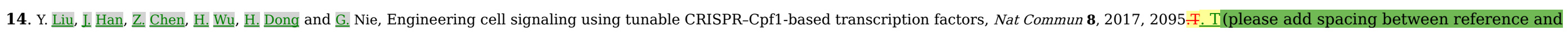

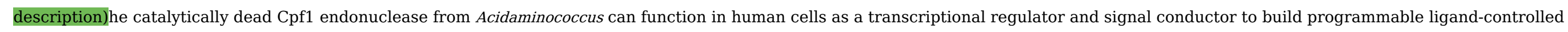
cell signaling circuits through the use of crRNAs with engineered riboswitches or G protein-coupled receptors linked to the dAsCpf1 protein.

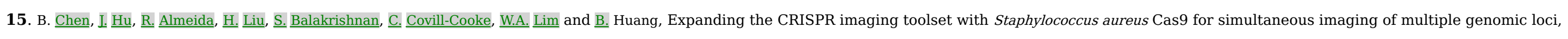
Nucleic Acids Res 44, 2016, e75.Engineered SpCas9 and SaCas9 proteins for multiplexed imaging of endogenous genomic sequences to resolve specific genomic loci.

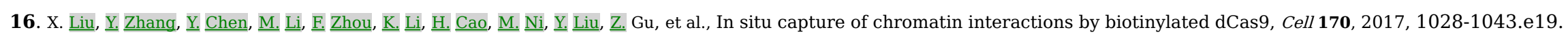

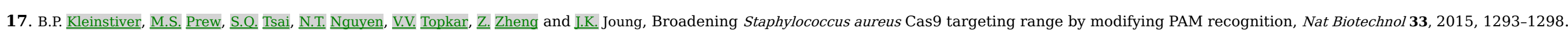

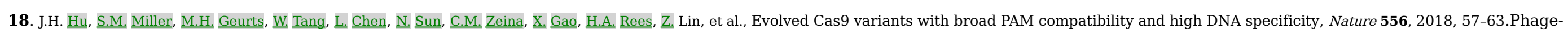

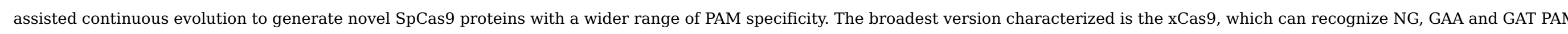
sequences between others, and shows greater DNA specificity compared to the SpCas9.

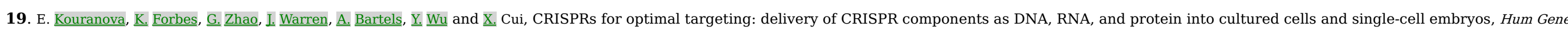
Ther 27, 2016, 464-475.

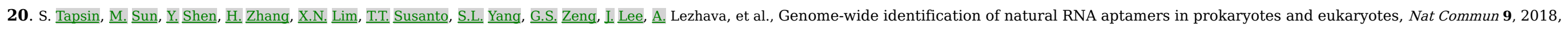

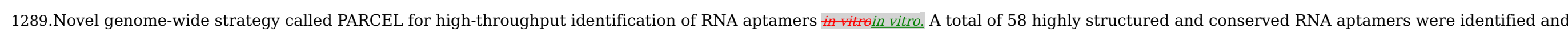
unexpectedly prevalent in coding regions.

21. M. Mandal and R.R. Breaker, Adenine riboswitches and gene activation by disruption of a transcription terminator, Nat Struct Mol Biol 11, $2004,29-35$.

22. R.T. Batey, S.D. Gilbert and R.K. Montange, Structure of a natural guanine-responsive riboswitch complexed with the metabolite hypoxanthine, Nature 432, $2004,411-415$.

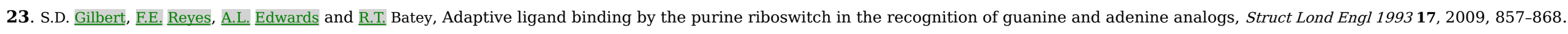

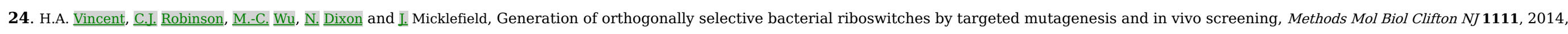
$107-129$.

25. P.J. McCown, I.J. Liang, Z. W. Weinberg and ․․‥ Breaker, Structural, functional, and taxonomic diversity of three PreQ1 riboswitch classes, Chem Biol 21, 2014, 880-889.

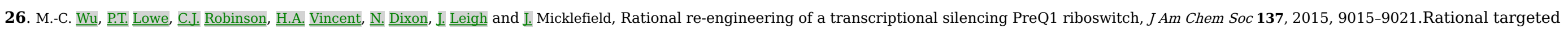
approach to re-engineer the PreQ1 riboswitch from Bacillus subtilis into an orthogonal OFF-switch.

27. E.R. Lee, K.F. Blount and ․R. Breaker, Roseoflavin is a natural antibacterial compound that binds to FMN riboswitches and regulates gene expression, RNA Biol 6, 2009, 187-194.

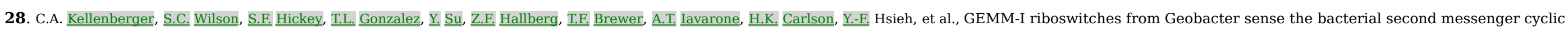


AMP-GMP, Proc Natl Acad Sci U S A 112, 2015, 5383-5388.

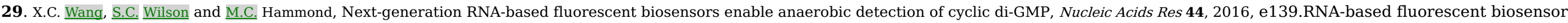
designs, selectively responding to cyclic di-GMP, to study host-microbial and microbial-microbial and microbial-microbial interactions via small molecule signaling.

30. J_. Ouellet, RNA fluorescence with light-up aptamers, Front Chem 4, 2016.

31. A. Autour, E. Westhof and M. Ryckelynck, iSpinach: a fluorogenic RNA aptamer optimized for in vitro applications, Nucleic Acids Res 44, 2016, 2491-2500.

32. C.C. Fowler, E.D. Brown and $\underline{\underline{Y} . \mathrm{Li}}$, Using a riboswitch sensor to examine coenzyme B12 metabolism and transport in E. coli, Chem Biol 17, 2010, 756-765.

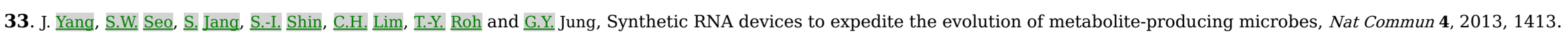

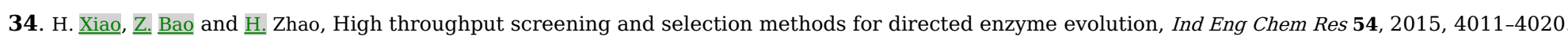

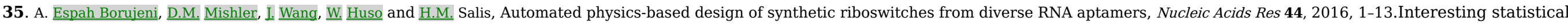
thermodynamic model to predict sequence-structure-function relationships of riboswitches for gene activation.

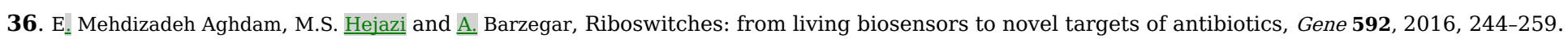

37. A.V. Sherwood, I.K. Frandsen, F.J. Grundy and T.M. Henkin, New tRNA contacts facilitate ligand binding in a Mycobacterium smegmatis T box riboswitch, Proc Natl Acad Sci U S A 2018,

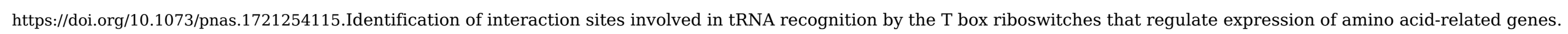

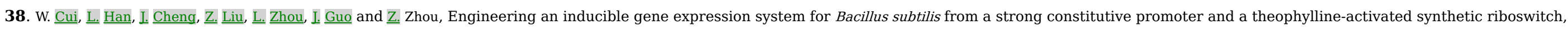
Microb Cell Factories 15, 2016.

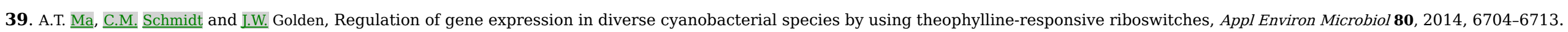

40. M.M. Rudolph, M.-P. Vockenhuber and B. Suess, Synthetic riboswitches for the conditional control of gene expression in Streptomyces coelicolor, Microbiology 159, $2013,1416-1422$.

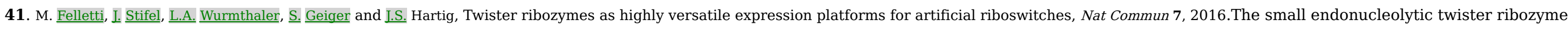

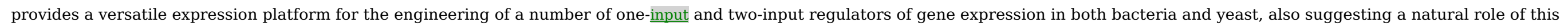
widespread ribozyme class.

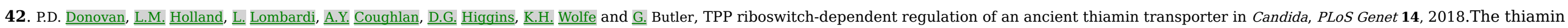

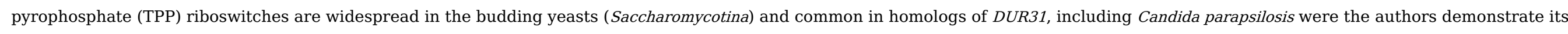
molecular function.

43. M.A. Moldovan, S.A. Petrova and M.S. Gelfand, Comparative genomic analysis of fungal TPP-riboswitches, Fungal Genet Biol 114, $2018,34-41$.

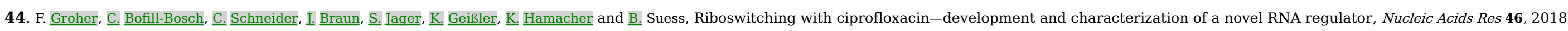
2121-2132.

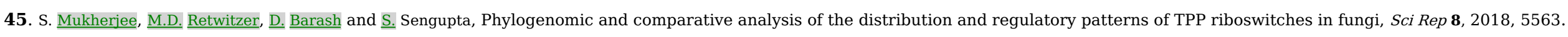

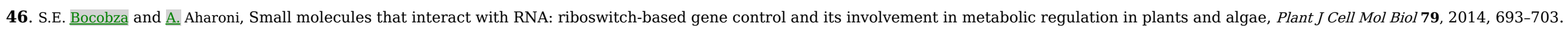

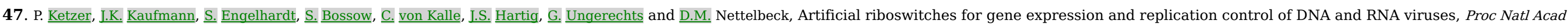
SCi U S A 111, 2014, E554-E562. 


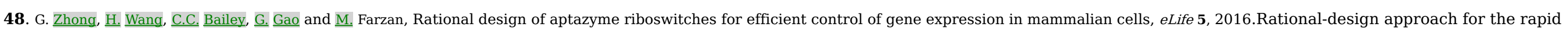

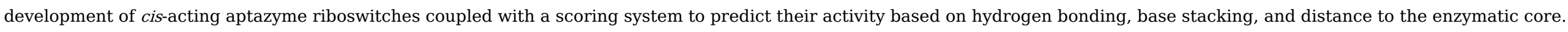

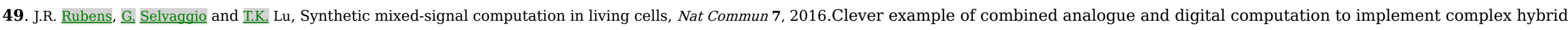
synthetic genetic circuits in living cells.

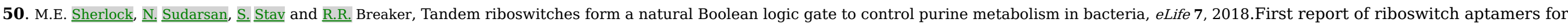

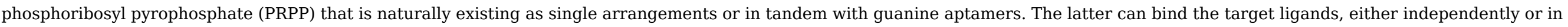

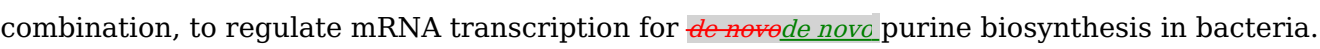

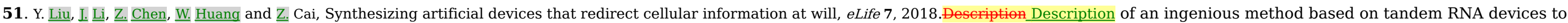

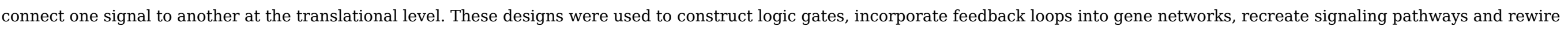
pre-existing such as the oncogenic signaling to reverse the malignant phenotypes of cancer cells.

52. Y. Liu, $\underline{\text { Y. Liu }}$ and $\underline{\text { M. }}$ Wang, Design, optimization and application of small molecule biosensor in metabolic engineering, Front Microbiol 8, 2017.

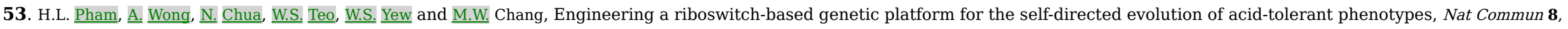

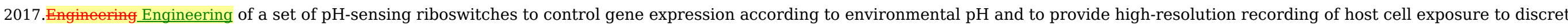

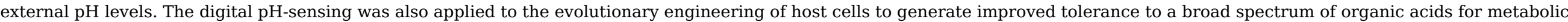
engineering and bioremediation applications.

54. J.C. Liang, A.L. Chang, A.B. Kennedy and C.D. Smolke, A high-throughput, quantitative cell-based screen for efficient tailoring of RNA device activity, Nucleic Acids Res 40, 2012 , e154.

55. L. Bastet, ․ Turcotte, I.T. Wade and D.A. Lafontaine, Maestro of regulation: riboswitches orchestrate gene expression at the levels of translation, transcription and mRNA decay, RNA Biol 2018, https://doi.org/10.1080/15476286.2018.1451721

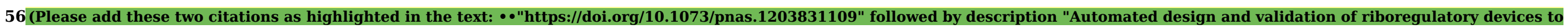

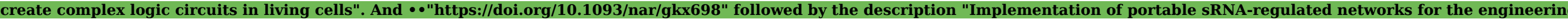

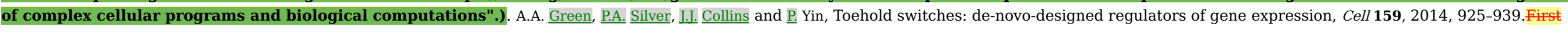

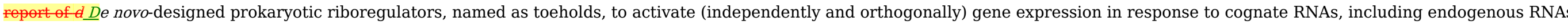

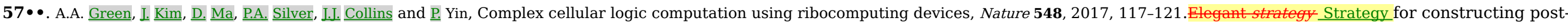
transcriptional RNA-only nanodevices to evaluate and compute complex logic in living cells through predictable and designable base-pairing rules.

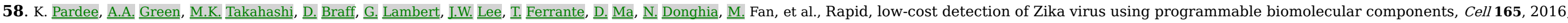

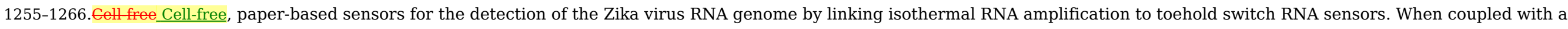
CRISPR/Cas9-based module, the sensor can discriminate between viral strains with single-base resolution.

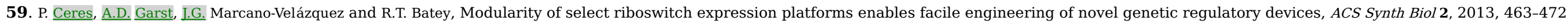

60. B. Suess and I.E. Weigand, Engineered riboswitches: overview, problems and trends, RNA Biol 5, 2008, 24-29.

61. A.D. Ellington and I.W. Szostak, In vitro selection of RNA molecules that bind specific ligands, Nature 346, 1990, 818-822.

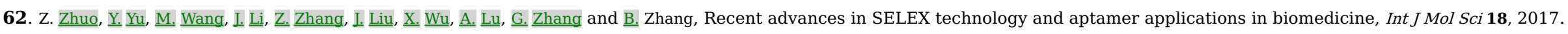

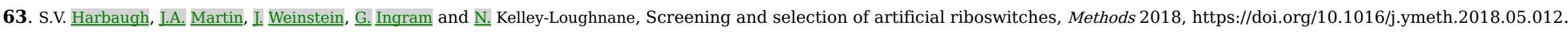




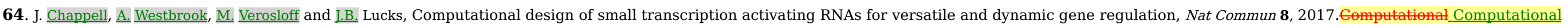

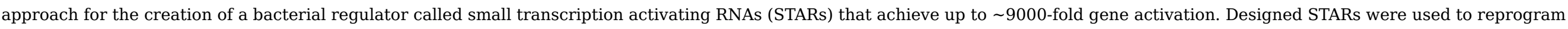
cellular phenotypes, to control multigenic metabolic pathways and in combination with CRISPRi.

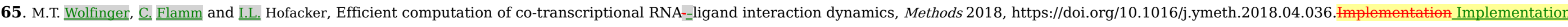
for computational analysis of co-transcriptional RNA-_ligand interaction dynamics to model concentration-dependent metabolite binding/unbinding kinetics.

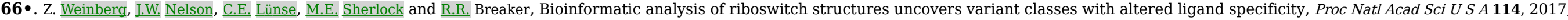

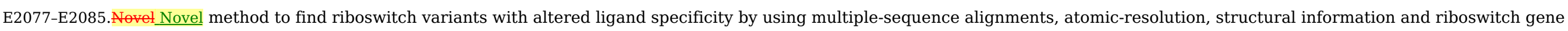
associations.

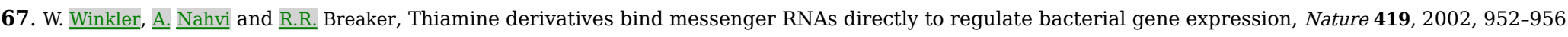

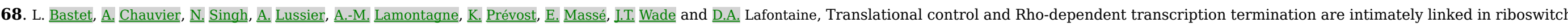

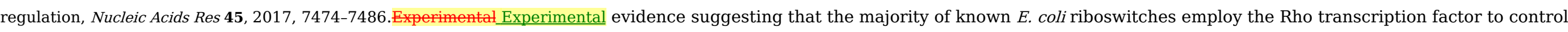
transcription termination and regulate mRNA levels.

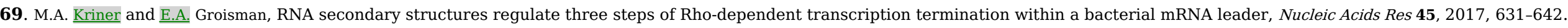

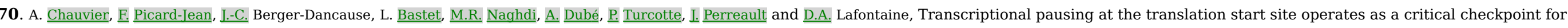

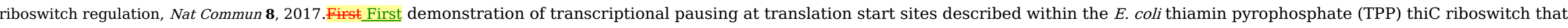
acts as a checkpoint for thiC gene regulation.

71. J.A. Collins, I. Irnov, S. Baker and W.C. Winkler, Mechanism of mRNA destabilization by the glmS ribozyme, Genes Dev 21, 2007, 3356-3368.

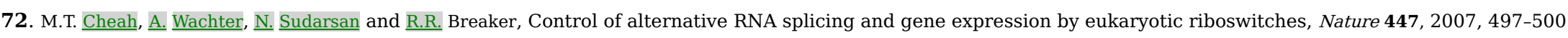

73. M.T. Croft, M. Moulin, M.E. Webb and A.G. Smith, Thiamine biosynthesis in algae is regulated by riboswitches, Proc Natl Acad Sci U S A 104, $2007,20770-20775$.

74. S. Bocobza, A. Adato, T. Mandel, ‥ Shapira, E. Nudler and A. Aharoni, Riboswitch-dependent gene regulation and its evolution in the plant kingdom, Genes Dev 21, 2007, 2874-2879.

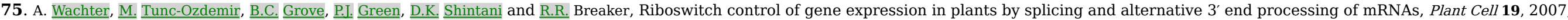
3437-3450.

76. J.R. Babendure, Control of mammalian translation by mRNA structure near caps, RNA 12, 2006, 851-861.

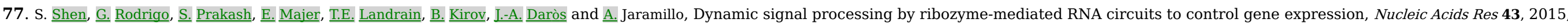

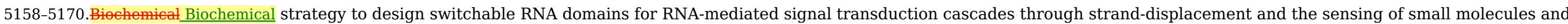
small RNAs.

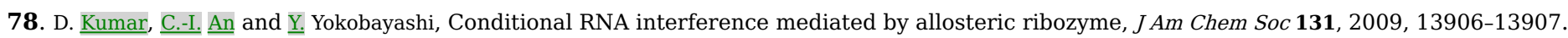

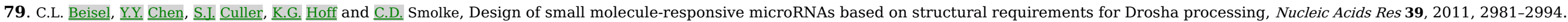

80. P. Machtel, $\underline{\underline{K} .}$ Bąkowska-Żywicka and $\mathrm{M} \geqq \_$Żywicki, Emerging applications of riboswitches-_from antibacterial targets to molecular tools, J Appl Genet 57, $2016,531-541$.

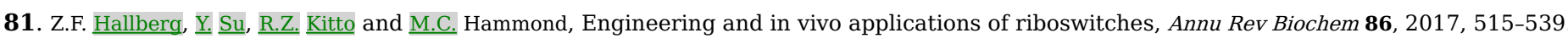

82. J. Chappell, K.E. Watters, M.K. Takahashi and J.B. Lucks, A renaissance in RNA synthetic biology: new mechanisms, applications and tools for the future, Curr Opin Chem Biol 28, 2015 , 47-56. 


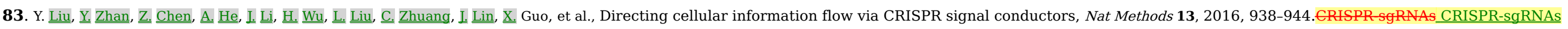
based riboswitches that recognize specific signals to regulate transcription of endogenous genes in response to external or internal signals of interest in mammalian cells.

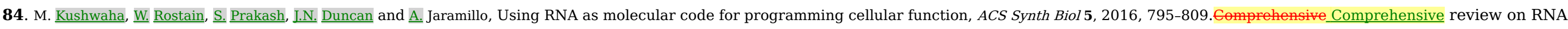
engineering and programmability including RNA parts, methods and computational models for rationally designed synthetic biology.

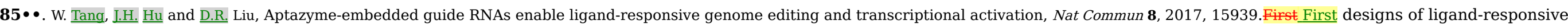
aptazymes incorporated into CRISPR-sgRNAs for small molecule-controlled genome editing and transcriptional activation in mammalian cells.

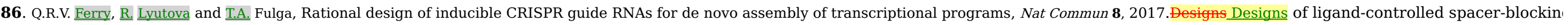

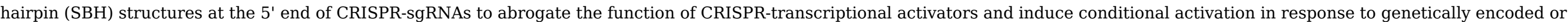
external triggers.

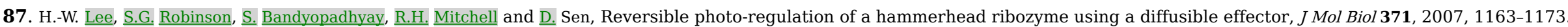

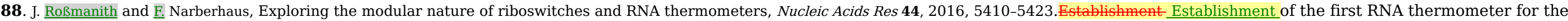
control of transcription.

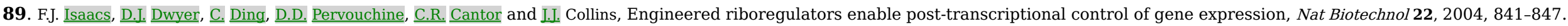

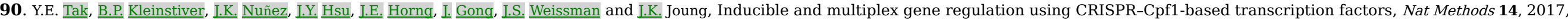
1163-1166. Engineered Engineered drug-inducible catalytically inactive Cpf1 nuclease for transcriptional activation of endogenous genes in human cells.

91. R.W. Bradley, M. Buck and B. Wang, Recognizing and engineering digital-like logic gates and switches in gene regulatory networks, Curr Opin Microbiol 33, $2016,74-82$.

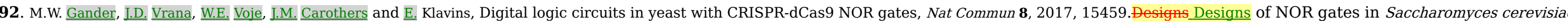
based on catalytically inactive dCas9 fused to the chromatin remodeller Mxi1 to construct the largest logic circuits to date.

93. A.M. Hammond and R. Galizi, Gene drives to fight malaria: current state and future directions, Pathog Glob Health 2018, https://doi.org/10.1080/20477724.2018.1438880.

94. P.D. $\underline{\text { Hsu, }}$ E.S. Lander and E. Zhang, Development and applications of CRISPR_-_Cas9 for genome engineering, Cell 157, 2014, 1262-1278.

95. H. Wang, M.L. Russa and L.S. Qi, CRISPR/Cas9 in genome editing and beyond, Annu Rev Biochem 85, 2016, 227-264.

96. R. Barrangou and I.A. Doudna, Applications of CRISPR technologies in research and beyond, Nat Biotechnol 34, 2016, 933-941.

97. R. Peng, G. Lin and I. Li, Potential pitfalls of CRISPR/Cas9-mediated genome editing, FEBS J 283, 2016, 1218-1231.

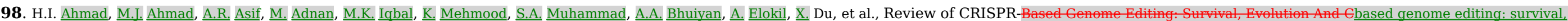
evolution and challenges, Curr Issues Mol Biol 2018, https://doi.org/10.21775/cimb.028.047(looks different format from other references as non of the others include doi:.... Please doublecheck.).

99. M! Adli, The CRISPR tool kit for genome editing and beyond, Nat Commun 9, 2018

\section{Graphical abstract}

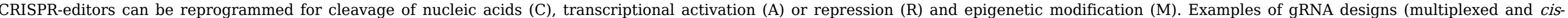
repressed interacting gRNA). Chemical or biological (proteins or RNAs) ligands for regulation. Computational design and testing of gRNA-_ligand interactions. 


\section{Highlights}

- Riboswitches and CRISPR-editing challenges, current and future applications.

- Riboswitches can be applied to sense CRISPR-sgRNAs.

- CRISPR gRNA riboswitches can function in all kingdoms of life.

- Combined in vivo screening and computational analysis to improve riboswitch regulation.

\section{Queries and Answers}

Query: The author names have been tagged as given names and surnames (surnames are highlighted in teal color). Please confirm if they have been identified correctly. Answer: Yes 